\title{
PLURALISMO JURÍDICO Y DEMOCRACIA EN MÉXICO. Entre el control de la diversidad y la ilusión de la igualdad
}

\author{
José Israel HERRERA \\ Universidad Autonoma de Campeche (México) \\ jiherrer@uacam.mx
}

LEGAL PLURALISM AND DEMOCRACY IN MEXICO. Between the control of diversity and the illusion of equality

Resumen: En este artículo se diserta sobre la admisión del pluralismo jurídico en México y la forma en la que las instituciones políticas han admitido o negado este concepto en México. Se presenta el concepto de pluralismo jurídico como un discurso más de tipo legal y de igualdad política de tipo artificial y controlada por el estado, que como una institución que opere bajo las condiciones y opciones reales de lo que el pluralismo en realidad es. Se hace un análisis de la forma en la que el pluralismo ha sido entendido y admitido en la legislación mexicana y en la actuación de la Suprema Corte de Justicia de la Nación. Asimismo, se presenta un análisis de la relación del pluralismo y su relación paradigmática con la democracia en la que el pluralismo pareciera ser un elemento a evitar antes que un elemento a promover.

Abstract: This article discusses the admission of legal pluralism in Mexico and the way in which political institutions have admitted or denied this concept in Mexico. The concept of legal pluralism is presented as a more legal discourse and political equality of an artificial and state controlled type than as an institution operating under the conditions and real options of what pluralism really is.

An analysis is made of the way in which pluralism has been understood and admitted in Mexican legislation and in the actions of the Supreme Court of Justice of the Nation. Likewise, an analysis of the relationship between pluralism and its paradigmatic relationship with democracy is presented, in which pluralism seems to be an element to be avoided rather than an element to be promoted.

Palabras clave: Pluralismo Jurídico. Democracia. Diversidad. México Legal Pluralism. Democracy. Diversity. Mexico 


\section{Introducción}

En los últimos años el término pluralismo jurídico se ha venido escuchando con mayor frecuencia no solo en el país sino en el mundo.

Los procesos de descolonización que experimentó Europa en los años 50’a y 60’s, el decenio internacional de los pueblos indígenas y sus movimientos sociales de reivindicación, las recomposiciones políticas en África, así como el fortalecimiento de sus sistemas de gobierno, la primavera árabe y el choque de civilizaciones, han ocasionado que escuchemos voces, veamos movimientos, así como soli0citudes que retan al estado en su estructura misma.

Los procesos mencionados, nos muestran la existencia de estados plurales, y de la existencia de sistemas normativos contenidos dentro de las fronteras de los estados, cuyo reconocimiento, análisis y conocimiento se ha convertido en un reto para los estados en estos años en todos los aspectos.

\section{¿Qué es el Pluralismo Jurídico?}

México desde el año de 1992 se ha definido como una Nación única e indivisible con una composición pluricultural. Previamente a ello las menciones a la igualdad, equidad o cualquier otro sinónimo eran prácticamente ausentes.

Las razones de esta reforma se encuentran en la ratificación del PIDESC y del PIDCYP ratificados por México en 1989, así como el que también se identifican presiones externas para la aprobación y ratificación del Convenio 169 de la Organización Internacional del Trabajo sobre pueblos indígenas y tribales asó como la preparación de la legislación mexicana como requisitos para la entrada en vigor del TLC (NAFTA) que establecía principios relacionados con Derechos Humanos, como requisitos adicionales para la firma. También "la aprobación de los “Acuerdos de San Andrés" y la posterior aprobación de lo se denominó la "Ley Indígena”". (Krotz 2001) (Krotz 2001: 2 - 3).

Todas estas reformas, políticas y legislativas, nos hablan de un proceso de reconocimiento como plurales, pero en el que no se sabe que tan plurales podemos ser o debíamos de ser.

Bobbio sobre el Pluralismo Jurídico señala "el Estado no es el único centro productor de normas jurídicas sino también el producido por los grupos sociales diferentes al Estado, siempre y cuando: determinen sus fines propios, establezcan los medios para llegar a esos fines, distribuyan funciones específicas de los individuos que componen el grupo para que cada uno colabore, a través de los medios previstos, para el logro del fin y que tengan diferente cultura" (Bobbio 2005). ${ }^{1}$

Entonces el Pluralismo Jurídico es la coexistencia dentro un Estado de diversos conjuntos de normas jurídicas positivas en un plano de igualdad, respeto y coordinación. Es la coexistencia de dos o más órdenes jurídicos en un mismo ámbito de tiempo y de espacio.

Andre Hoekema divide al pluralismo jurídico formal, como de tipo igualitario o de tipo unitario. Según Hoekema, "el pluralismo jurídico de tipo unitario mantiene una relación de subordinación entre el Estado con su derecho nacional y el de otros sistemas, por lo que la coexistencia de dos o más sistemas de derecho en su sentido social ha sido reconocida por el derecho estatal, incluso en la misma Constitución, pero el derecho oficial se reserva la facultad de determinar unilateralmente la legitimidad y el ámbito de aplicación de los demás sistemas de derecho reconocidos"2 (Hoekema 2002). El pluralismo jurídico formal

$15^{\text {a }}$ Reimp., 2005, paginas $\left.10-13\right)$.

2 (Hoekema, 2002: 70) 
igualitario, "reconoce la existencia de comunidades distintas de índole étnica dentro de la sociedad nacional que, como tal tienen el derecho de desarrollar su propio sistema de instituciones, entre otros su derecho propio, como una parte diferente, pero de igual valor al orden político-legal del país"3 (Hoekema 2002). ${ }^{4}$

El pluralismo jurídico implica un reto para el estado, algunos de estos han respondido mediante principios como el de "Acomodación Razonable"5 planteado en Europa, en países mas de corte liberal, como Alemania, Holanda, países nórdicos, que plantean la posibilidad de coexistencia de los grupos que soliciten y vivan en esta sociedad o sociedades.

De hecho, este principio llevado a un nivel más alto, lo puedo denominar como un Principio de Máxima Acomodación, en el que es posible este acomodo al ser la igualdad un principio general y la existencia de la diversidad en una movilidad constante, siendo la legislación un producto que lejos de impedirle promueva la existencia de sociedades diversas y plurales en condiciones de igualdad.

\section{El conflicto del concepto Pluralismo Jurídico con la teoría del estado}

De acuerdo con Norberto Bobbio, el pluralismo nace del derecho de asociación: este es el punto. "La sociedad pluralista nace del hecho que se forman poco a poco en una sociedad democrática grupos de interés, sindicatos, partidos que aprovechan el derecho de asociación para acoger a personas que tienen los mismos intereses (sindicatos) o las mismas idea o ideologías (partidos) y que después terminan por transformarse en los verdaderos sujetos políticos de una sociedad democrática. En una sociedad avanzada son más sujetos políticos estos organismos, que son justamente asociaciones espontáneas y convencionales, que los sujetos aislados. Esto es, los individuos para contar políticamente deben pasar a través de estos grupos. En otras palabras, cuando se habla de democracia pluralista, se habla no tanto de democracia de muchos individuos: se habla de una democracia de muchos grupos."(Bobbio 1986)

La existencia de una diversidad en el estado, cualquiera que sea su expresión, o para el caso que nos ocupa de diferentes sistemas plurales, es una idea que causa un conflicto en la forma en la que se plantea. Esta puede ser admitida, admitida parcialmente, rechazada entre otras posibilidades.

El pluralismo puede ser visto como un enemigo de la estabilidad del estado. Schmitt por ejemplo designa con el término "homogeneidad" ese carácter sustancial de la identidad democrática. Si la democracia es esta capacidad de agrupar y defender una forma de vida ligada a una identidad sustancial, aquéllos que sean heterogéneos y no formen parte de ella, serán considerados enemigos" (Rossi 2014) a lo que sumaría yo traidores, minorías, populistas, o catalogados dentro de alguna categoría que signifique peligro o discordancia con el estado.

El miedo a una balcanización o desmembración del estado mexicano, ha estado presente en las discusiones sobre la admisión y reconocimiento a derechos territoriales a los pueblos y grupos indígenas del país. De ahí que haya influido ampliamente en el reconocimiento

3 (Hoekema, 2002: 76)

4 Hoekema André (2002), "Hacia un pluralismo jurídico formal de tipo igualitario”, El Otro Derecho, núm. 26-27, pp. 63-98

5 Esta doctrina ("raisonable accomodation") aparece, por primera vez, en la jurisprudencia norteamericana en el caso Sherbert (1963) (2), como una regla interpretativa de la Primera Enmienda constitucional que, como es bien sabido, se expresa en dos cláusulas: a) "establishment clause": prohibición de implantar una religión oficial en la Unión, y b) "Free exercice clause": libre ejercicio de la libertad religiosa y otras libertades fundamentales. Se propone como una vía para resolver conflictos entre leyes estatales (o normativas empresariales) y derechos fundamentales originados por demandas de ciudadanos o instituciones que entienden vulnerada su libertad religiosa y/o de conciencia en el ámbito laboral. (Díez 2016) 
de ciertos derechos territoriales o bien incluso en la limitación de los grupos que pueden seleccionar autoridades bajo sistemas tradicionales.

El control que el estado ejerce de manera fáctica sobre los grupos implicaría cambios políticos y legales profundos que cimbrarían las relaciones de poder, económicas con resultados de afectación a los propios grupos del poder. Postura que Galli deja entrever cuando señala que "La neutralización provocada por la soberanía, que caracteriza al Estado moderno, esconde en su núcleo más íntimo a la antipolítica: en ella aparece "la utopía de un orden unitario carente de política, esto es, que habría cancelado el poder, las partes "\%" (Rossi 2014).

De hecho, existe un paradigma que se puede ver no solo en la teoría de Galli, sino de otros autores consistente en el que, si tomamos en cuenta que existe una utopía de un orden unitario carente de política, implicaría dentro de una de las posibilidades, la cancelación misma del pluralismo jurídico al cancelarse las partes. Esto significa que una de sus posibilidades podría ser el tender hacia la cancelación del derecho a la diferencia. Esto implicaría que la política carecería del "polis", y pasaría de ser una política plural o del grupo sino un solo pensamiento o una "atomitica" del átomo o unidad o del "Individuus".

La idea de la participación plural por parte de Bobbio, Habermas o en Galli entre muchos otros no resuelve tampoco el dilema del reconocimiento. El proceso de decisión y participación son perfectos. Incluso la aplicación de una tercera vía habermasiana es posible ya que exige de forma obligatoria una participación política plural y amplia. Pero ninguna resuelve de forma efectiva, la pregunta del ¿Cómo esas partes llegan a serlo?

No existe problema con el individuo per se. El individuo es la esencia de la democracia. Galli señala que no es el grupo el que inicia el proceso sino el individuo. Actualmente basta solo el ser para poder participar en la democracia. Esto refleja un problema de la democracia y la teoría política y que consiste en el ¿Qué grupos son admitidos para participar en ella, cuáles son los requisitos procedimentales que se exigen, si es que hay una vía formal, y cuáles grupos no son reconocidos.

Constitucionalmente hablando hemos incorporado a la Constitución Política de los Estados Unidos Mexicanos derechos para la participación política de los grupos indígenas. Pero no estamos seguros de cuantos grupos indígenas lo son, ni si todos los grupos ya están reconocidos. Un dato de esto consiste que en los años 90's del siglo pasado se hablaban de 50 etnias. A inicios del siglo XXI, se hablaba de 62 y actualmente de 67.

Descubrimos una diversidad lingüística al crear el Instituto Nacional de Lenguas Indígenas (DOF 13/03/2003) por ejemplo. Pero quedan a un lado los derechos de grandes grupos que son más extensos en el país como son los menonitas o los asentamientos que se encuentran en Campeche provenientes de los campos de refugiados de Guatemala. Somos zona de paso de decenas de miles de indocumentados, muchos de los cuales se quedan a vivir en el país, algunos desde más de medio siglo y no conocemos el número exacto de etnias que hay en el país. No puedo participar como colonia menonita y gobernarme en usos y costumbres porque no tengo el reconocimiento formal de una autoridad estatal. Es más, ni siquiera tengo el status de comunidad. Nuestro problema no es menor.

6 Los textos de Galli sondean esta perturbación creciente y hallan una "contestación rabiosa”, pasiva y apática, unida a la aceptación resignada de que no hay alternativas a la democracia. El problema de un descontento semejante es su falta de productividad: la desafección y extrañeza respecto de la democracia se vuelven constantes, sin que surjan otras posibilidades disparadas por el nuevo estado de cosas. Este descontento implica un inconformismo respecto de la democracia que es simultáneamente conformista, ya que se resigna a la necesidad al mismo tiempo que reniega de ella. Galli utiliza una imagen para exponer la fuente de ese malestar: la democracia contemporánea se asemeja a un supermercado de derechos en el que se descubriera que las mercancías (los derechos) no están, sustituidas por consignas que sin embargo las anuncian y las proclaman ya presentes, de allí se deriva una anomia que se vuelve una disposición cada vez más aguda. adecuación de los ciudadanos bajo la forma de la resignación (Rossi 2014). 


\section{La democracia y el peligro de lo plural}

Bobbio sobre la democracia da una definición mínima para su entendimiento, al señalar que esta es "un conjunto de reglas que establecen que todos deben tomar parte en la decisión y la decisión debe ser tomada naturalmente después de un debate, por unanimidad o por mayoría", nos deja entrever que a libertad de entrada es un concepto peligroso para la existencia del estado mismo, o bien, se vive bajo un esquema de libertad, pero bajo los principios reconocidos ese estado como tales (Bobbio 1986).

Para Giovanni Sartori, la democracia es desde siempre y ante todo una forma de Estado y de gobierno, y esa sigue siendo la acepción primaria del término; en ese sentido, es un mecanismo de toma de decisiones públicas vinculantes y susceptibles de ser impuestas mediante la coacción del Estado (Sahuí Maldonado 2016).

No me refiero en este punto a lo que Galli denominaba un "callejón sin salida" derivado de la "complejidad de la democracia", de modo que las múltiples posibilidades escondidas en sus pliegues problematicen la necesidad que hoy impera...la persecución de un Nosotros inventado, una seudocomunidad en busca de una política igualitaria, el populismo se vuelve instrumento de una política que en realidad es aún más distante del pueblo de lo que lo era la política tradicional", es decir, por qué no aceptar que en ambos casos nos encontramos con un pueblo imaginario y mítico cuya función movilizadora tiene lugar en ambos contextos y con un valor antiinstitucional análogo (Rossi 2014).

La libertad, la igualdad y lo plural y diferente son conceptos adaptados a cada circunstancia. Esto nos lleva a reconocer que la diferencia se legisla bajo la figura de igualdad o la de diferencia per se. En términos de estado, el pluralismo jurídico ha tenido que ser un elemento que inicia estando alejado de las políticas del estado y su admisión, es más un triunfo de los sectores que buscan un espacio de participación o de reconocimiento entre otros.

La construcción y administración del pluralismo jurídico, su administración y su reconocimiento, puede ser hallada en diversos momentos en la legislación mexicana.

\section{Cultura media nacional}

Uno de los ejemplos más claros de las formas en las que la diferencia cultural fue administrada se encontraba en el artículo 220bis del Código Federal de Procedimientos Penales cuando hablaba de "cultura media nacional":

"Cuando el inculpado pertenezca a un grupo étnico indígena, se procurará allegarse dictámenes periciales, a fin de que el juzgador ahonde en el conocimiento de su personalidad y capte su diferencia cultural respecto a la cultura media nacional."

Los jueces en la utilización de este artículo señalaban que los indígenas juzgados presentan una diferencia cultural después de haberlos comparado con la cultura media nacional. Se decía en los expedientes que la cultura a la que pertenecen los indígenas es la maya, ya que las personas hablan la lengua maya, que tienen las costumbres de la región, que presentan un grado de escolaridad bajo, que trabajan en el campo, que cosechan productos de la región, que viven en casas mayas. Es decir, cuando se dicta sentencia, el juez suma características de lo que consideran "lo maya" en la región?. Mientras más características se puedan sumar, mayores posibilidades hay de que sea perteneciente a esta etnia. ${ }^{8}$

7 Esta concepción, de ir sumando características, nos acerca al empleo del término cultura de E. Tylor "aquel todo complejo que incluye el conocimiento, las creencias, el arte, la moral, el derecho, las costumbres y cualesquiera otros hábitos y capacidades adquiridos por el hombre en cuanto miembro de la sociedad."(Tylor 1979) Tylor, Edward, "La ciencia de la cultura" en Kahn, J. S. comp., El concepto de cultura: textos fundamentales, Anagrama, Barcelona, 1979, pp. 29 - 46.

8 Un ejemplo de su utilización lo encontramos en el siguiente caso. Un abogado defensor en uno de los expedi- 
Ahora bien, estos tres conceptos, cultura, nación y media hacen referencia a que es posible establecer cuáles son los elementos o características que integran a una nación, determinar los elementos que se establecen como mexicanos, a estos ubicarles un punto intermedio "la media", y con base a este lugar medio, se obtiene un punto de partida para comparar a una persona y saber si es indígena o no. Entonces, si comparamos la cultura de la persona que se piensa puede ser indígena, con lo que el México imaginario cree es la cultura media nacional, este proceso de comparación ofrece por lo tanto dos resultados: 1. Encontrarse dentro de lo que se ha establecido como cultura media nacional, 2. Encontrarse fuera de la cultura media nacional. Si la cultura comparada se encuentra dentro de la cultura media nacional, es por lo tanto "mexicano" y no podría ser juzgado como "indígena".

La segunda opción consiste en que la persona comparada, se encuentre fuera de la cultura media nacional. Si la persona se localiza en esta situación, existen dos posibilidades lógicas: primero, que se encuentre fuera por ser indígena o, segundo, que se encuentre fuera por no ser ni indígena ni mexicano o sea un extranjero.

Es decir, el sistema judicial mexicano, una vez que concluía el proceso de comparación, al determinar que la persona es indígena, le excluye por no tener las características de lo que se considera mexicano, pero, le juzga bajo los criterios nacionales. Le excluía para poder juzgarle, como si fuera un mexicano. ${ }^{9}$ Se llega a la conclusión de que el indígena no se encuentra dentro de la categoría de lo que es mexicano, pero se le juzga como si lo fuera, bajo todas las reglas del México Imaginario que identificaba Bonfil.

La cultura media nacional, se reduce entonces a un término que hace referencia a una ecuación matemática que consiste en: el punto intermedio de la suma de los rasgos previamente considerados como mexicanos.

\section{El pluralismo, la igualdad y la CPEUM}

El pluralismo jurídico se encuentra íntimamente ligado al concepto de igualdad, no necesariamente opuestos, sino que son espacios en los que hay diálogos para su entendimiento y/o confrontación.

La igualdad (de ningún tipo) no se encontraba garantizada en la Constitución Política de los Estados Mexicanos, menos aún el concepto de pluralismo. "El derecho a la igualdad, en tanto cláusula de reconocimiento, ha ganado terreno en la Constitución"10 (Rossi 2014).

La constitución mexicana ha hecho 3 reformas sobre igualdad y una relativa a pluralismo jurídico. La primera de ellas se dio en el año de 1947 cuando se expidió el Decreto que

entes señala: en su declaración preparatoria mi defendido negó estar caminando por la calle principal de Dzonot Carretero y manifestó que si anteriormente afirmó que fue detenido sobre la calle principal de la localidad fue porque le dijeron por una persona de las que estaban en la camioneta que le detuvo donde fue trasladado hasta Progreso, Yucatán, que así lo hiciera para que no tuviera problema y saliera en libertad rápida manifestándole que solo sería acreedor de una multa prometiéndole que le darían facilidades para regresar a su pueblo, lo que es creíble tomando en consideración, la calidad de campesino, la condición de indígena y la diferencia cultural con respecto a la media nacional de mi defendido (Expediente 481/2002-A Tribunal Unitario del $14^{\circ}$ Distrito. Página 15). Posteriormente el abogado defensor continúa: en el dictamen en antropología social, se dictamina que las costumbres son las mismas de la etnia maya de Dzonot Carretero, que presenta diferencia cultural respecto a la cultura media nacional, que no consideran reprochable la portación de un arma de fuego para las labores del campo, y que valorando la diferencia cultural existente ésta le impidió en forma insuperable estimar delictuosa la conducta que se le imputa consistente en portar un arma de fuego sin licencia (Expediente 481/2002-A Tribunal Unitario del $14^{\circ}$ Distrito. Página 16).

$9 \mathrm{El}$ proceso de comparación que plantea la legislación mexicana, ubica al indígena como un ser que se encuentra fuera de lo que es considerado mexicano.

10 En efecto, el trayecto de la igualdad en la Constitución mexicana no puede entenderse como uno de mera ampliación de su contenido y alcance, sino como uno que ha privilegiado a la igualdad como reconocimiento y a la igualdad como representación política, pero que ha demeritado a la igualdad como redistribución.” p. 309 libro 100 ensayos 
adiciona el párrafo primero de la fracción primera del artículo 115 de la Constitución Política de los Estados Unidos Mexicanos que Incorpora la participación de las mujeres en las elecciones municipales, en igualdad de condición que los varones, con el derecho de votar y ser votadas el día 12 de febrero de 1947.

El 31 de diciembre de 1974 se expidió el Decreto que reforma y adiciona los Artículos 4o., 5o., 30 y 123 de la Constitución Política de los Estados Unidos Mexicanos, en relación con la Igualdad Jurídica de la Mujer. Incorpora el principio de que el varón y la mujer son iguales ante la ley. Establece bases constitucionales para permitir igualdad jurídica de las mujeres en materia de ejercicio de profesiones, derechos laborales y seguridad social.

Posteriormente, el 11 de diciembre de 1988 tocó turno al Decreto por el que se reforma la Fracción X del Artículo 89 de la Constitución Política de los Estados Unidos Mexicanos. Precisa la facultad del Presidente de la República para dirigir la política exterior y celebrar tratados internacionales, con la aprobación del Senado. Incluye los siguientes principios para la conducción de la política exterior: la autodeterminación de los pueblos; la no intervención; la solución pacífica de controversias; la proscripción de la amenaza o el uso de la fuerza en las relaciones internacionales; la igualdad jurídica de los estados; la cooperación internacional para el desarrollo; y la lucha por la paz y la seguridad internacionales.

Y finamente el 22 de mayo de 2015 se expidió el Decreto por el que se reforma la fracción III, del Apartado A, del artículo 2o. de la Constitución Política de los Estados Unidos Mexicanos. Determina la garantía de que "las mujeres y los hombres indígenas disfrutarán y ejercerán su derecho de votar y ser votados en condiciones de igualdad; así como a acceder y desempeñar los cargos públicos y de elección popular para los que hayan sido electos o designados"; e incorpora que "en ningún caso las prácticas comunitarias podrán limitar los derechos político electorales de los y las ciudadanas en la elección de sus autoridades municipales".

La mención al pluriculturalismo se dio por primera vez con el Decreto por el que se reformó el Artículo 4o. de la Constitución Política de los Estados Unidos Mexicanos 28 de enero de 1992 con el que se incorpora en la Constitución el principio de que la Nación mexicana tiene una composición pluricultural sustentada originalmente en sus pueblos indígenas. Establece que la ley secundaria protegerá y promoverá el desarrollo de sus lenguas, culturas, usos, costumbres, recursos y formas específicas de organización social, y garantizará a sus integrantes el efectivo acceso a la jurisdicción del Estado.

Contrariamente a lo que se puede considerar, las admisiones a estos derechos han sido en una gran medida recientes y no sin ser polémicas y sobre todo algunas de estas controvertidas como la "reforma indígena".

\section{El pluralismo jurídico y la SCJN}

Robert Alexy ha indicado que los tribunales pueden contribuir a mejorar los procesos políticos en las democracias constitucionales potenciando la voz de las minorías. "Quien consiga convertir en vinculante su interpretación de los derechos -esto es, en la práctica, quien logre que sea la adoptada por el Tribunal Constitucional Federal- habrá alcanzado lo inalcanzable a través del procedimiento político usual" (Alexy, 2009: 36) (Sahuí Maldonado 2016).

La constitución como depositaria de los conceptos jurídicos elementales, no se ha visto exenta de presentar una interpretación sobre el sentido de conceptos como el de igualdad y pluralismo. Las interpretaciones que generaron durante la novena época no han sido abundantes. ${ }^{11}$

11 La igualdad no sólo es lo que expresa el texto constitucional, sino sobre todo es la interpretación que se ha hecho de ese principio y derecho. Por su propia evolución en la Constitución, inicialmente los criterios interpretativos no fueron muchos, hasta la novena época. (Serrano 2017) 
Aunque existen un par de interpretaciones a destacar como son el amparo en revisión 1174/99, sobre los límites de la igualdad formal, en el que se señaló que la igualdad "ha de servir de criterio básico para la producción normativa y su posterior interpretación y aplicación..." por lo que "si bien es cierto que el verdadero sentido de la igualdad es colocar a los particulares en condiciones de poder acceder a derechos reconocidos constitucionalmente, lo que implica eliminar situaciones de desigualdad manifiesta, ello no significa que todos los individuos deban ser iguales en todo".

El amparo directo en revisión 988/2004 señaló que el derecho a la igualdad es inherente a las personas y que "debe entenderse como un principio que exige tratar igual a los iguales y desigual a los desiguales; (y que) para ajustarse a ello, en algunas ocasiones hacer distinciones estará vedado, pero en otras estará permitido, o incluso constitucionalmente exigido", lo que a su vez tiene como consecuencia que la igualdad sea "un principio complejo que otorga a las personas no solamente la garantía de que serán iguales ante la ley sino también en la ley.

La Décima Época, iniciada en junio de 2011 ha emitido alrededor "de 100 sentencias vinculadas con la igualdad en todos sus aspectos. Asimismo, se han emitido más de 150 tesis (aisladas y jurisprudenciales). Una de suma importancia fue cuando la la SCJN pasó a distinguir la igualdad formal de la de hecho y con ello, abrió paso a la protección de grupos sociales históricamente desaventajados (Serrano 2017).

El Pleno adoptó el criterio en la Acción de Inconstitucionalidad 08/2014, en la que describe un racismo para ciertos grupos provocado por la aparente neutralidad del ordenamiento emitido. "Para explicar esta discriminación por resultado, el Pleno de la Corte desarrolla la idea de la discriminación estructural ${ }^{12}$ para exponer la forma en que ésta sustenta la producción e interpretación normativa (Serrano 2017) .

Esto no es ajeno a lo que Morlino afirma sobre los dos estadios en la afirmación del valor igualdad. El primero de ellos -el más aceptado según el autor- concierne a la igualdad formal frente a la ley y se traduce en la prohibición de discriminaciones por razón de sexo, raza, etnia, religión, etcétera, mientras que el segundo -más distintivo, si lo que se quiere conceptualizar es una dimensión singular distinta a la procedimental del imperio de la ley-, tiene que ver con la remoción de los obstáculos que limitan la igualdad social y económica y, por lo tanto afectan el desarrollo pleno de la persona y la efectiva participación de los trabajadores. De modo típico el autor piensa la igualdad como un valor material, sustantivo, asociado a la solidaridad y a los derechos sociales y económicos, y subraya la dificultad de garantizarlos debido a sus altos costos (Ibíd., 2005a: 280-281) (Sahuí Maldonado 2016) (SAHUÍ 2016 P. 282-283).

Ahora si bien se puede afirmar que el criterio de la SCJN "representa un gran avance en la protección del derecho a la igualdad, pues reconoce la existencia de una estructura de dominación en contra de ciertos grupos sociales y que tiene el potencial de determinar el sentido de las normas, con o sin intención de por medio" (Serrano 2017), esto no significa que se haya hecho hasta el momento un balance para determinar cuáles son las normatividades que se encuentran potencialmente en esta situación. Dentro de estas se encuentran aquellas relacionadas con la participación política de los grupos y de las comunidades minoritarias que como he señalado no tienen el poder, o no se han empoderado o incluso a las que no se les permite el ser parte del diálogo y la toma de decisiones en el país. Podríamos señalar que existe un racismo, aunque admitido, estructuralmente consentido.

\section{Consideraciones finales}

De lo que se ha discutido entonces nos podemos preguntar ¿Cuál es la relación entre pluralismo jurídico y democracia?

12 En este capítulo se habló por ejemplo de la cultura media nacional que era altamente discriminatoria aun cuando su función parecía ser beneficiosa. 
El pluralismo ha sido identificado en las democracias como un elemento inherente a la sociedad misma. Galli señala que "No existe una sociedad democrática con un pensamiento único o sin pensamiento" (Galli 2013).

De entrada, la función misma de una democracia consiste en conciliar diversos intereses y opiniones provenientes no solo de un individuo sino de colectividades agrupadas bajo varias denominaciones. ${ }^{13}$

La democracia deliberativa no se reduce al diseño de requisitos procedimentales, sino que se ofrece como una reinterpretación de una intuición democrática básica, a saber: que las decisiones políticas son legítimas y, por tanto, vinculantes tan sólo en la medida en que sean resultados de procesos deliberativos colectivos en los que hayan participado todos aquellos a quienes van dirigidas (Habermas 1998, 363-406 en Velasco 2009).

No cabe duda de que todo órgano colectivo de deliberación y de toma de decisiones colectivas tendrá por fuerza que llegar a acuerdos que de modo circunstancial excluirán algunos intereses y pretensiones individuales o grupales. Dado que la regla de mayoría es el criterio decisorio fundamental de la democracia, todas las posiciones perdedoras son minoritarias por definición. Como dice Sartori, la regla de la mayoría "fabrica" a la minoría. Sin embargo, cuando tales posiciones están esencialmente atadas a identidades particulares sobre las que pesa un prejuicio o estigma social, la institución democrática deviene perversa porque entonces reproduce y no subvierte las estructuras de inequidad (Sahuí Maldonado 2016).

La defensa de las minorías democráticas, empero, no conduce necesariamente a pensar su participación o representación mediante mecanismos determinados como la representación proporcional o las cuotas; también serían admisibles medios jurisdiccionales para hacerse escuchar y exigir al aparato de gobierno respuestas fundadas y motivadas en derecho (Sahuí Maldonado 2016).

Sin embargo, tanto Bobbio, Galli, o Habermas, nos explican la democracia entre autores ya instaurados y no desde los emergentes, aquellos grupos que exigen una voz y participación no bajo los términos siempre estatales, sino bajo los suyos propios.

La democracia nos presenta varios retos estructurales. ¿Cómo podemos dialogar entre grupos y sectores que no poseen los mismos medios de participación? $\mathrm{O}$ ¿entre grupos desiguales y políticamente asimétricos?

Por ejemplo, en el caso de los jueces de Quintana Roo, inventamos una estructura judicial, que al final después de varias décadas será llamada oficial, ya que se habrá implantado en el imaginario colectivo de forma profunda. En el caso de una ley que mide a las personas dentro de un parámetro llamado media nacional, solo refleja la existencia de un etnocentrismo que busca crear a las personas bajo lo que Bartra llamaba el Salvaje ante el espejo.

A través del concepto de "poder comunicativo" entre los ciudadanos, la "tercera vía" de Habermas valora el momento de decisión de la soberanía popular. O sea, el momento fundamental para cualquier democracia, el cómo se delibera. Necesitamos profundizar en estos diálogos, pero ya no entre los grupos que han sido admitidos, sino entre los grupos que aún están por ser admitidos y a los cuales hemos quitado visibilidad ante la no admisión de los mismos.

Debido a ello, en muchas ocasiones se habla más del órgano garante de las elecciones que del ciudadano mismo, se blinda un proceso electoral con procesos repetitivos e impuestos, y al ciudadano se le visualiza como el votante que debe seleccionar. Medimos el éxito de una elección por el número de votantes, una elección es un éxito si hay una gran afluen-

13 La emisión del voto representa únicamente el momento final del proceso democrático, de modo que la calidad de una democracia no se mide por el número de votos emitidos, sino por las condiciones del proceso previo a la votación, del proceso de formación de la opinión sobre la que se basa el voto, del hecho de que cada ciudadano haya contrastado sus propias preferencias con las de los demás, de que haya corregido sus propios juicios tras recibir nueva información y de que haya intentado ponderarlas a la luz de razones imparciales, a lo que agregaría entre los diversos grupos. (Velasco 2009). 
cia. Se generan elecciones sin que haya un análisis previo, o un conocimiento del político, sin que haya una deliberación.

La relación entre pluralismo y democracia es aún un debate inacabado, que se verá fortalecido ante la aparición, causada o no, de nuevos grupos, el descubrimiento de los mismos, o la autorización a su participación. Quizás debemos de comenzar a pensar en el número de actores no individuales que participan. En el número de sectores que son incluidos. Si bien un pluralismo jurídico total, podría ser imposible. Pero eso no implica que no se deba de dejar de reconocer que el pluralismo es una realidad en todos los niveles de la sociedad, en la que aún existen sectores y actores que aún no han sido tomados en cuenta y lejos aún son excluidos estructuralmente por la legislación.

\section{Bibliografía}

Bobbio, Norberto (1986). “Democracia Y Pluralismo.” Revista de Ciencia Política VIII (1-2): 12737.

Bobbio, Norberto (2005). Teoría General Del Derecho. 5thed. Bogotá: Temis.

Díez, José Antonio (2016). "El Principio de La 'razonable Acomodación' en Los Conflictos Sobre Discriminación Laboral." UNIR Revista. http://www.unir.net/derecho/revista/noticias/ el-principio-de-la-razonable-acomodacion-en-los-conflictos-sobre-discriminacion-labo$\mathrm{ral} / 549201458365 /$.

Galli, Carlo (2013). El Malestar de La Democracia. Buenos Aires: Fondo de Cultura Económica.

Hoekema, Andre (2002). "Hacia Un Pluralismo Jurídico Formal de Tipo Igualitario." El Otro Derecho, no. 26-27: 63-98.

Krotz, Esteban (2001). “Antropología Jurídica Y Cultura Maya Actual.” In Aproximaxiones a La Antropología Jurídica de Los Mayas Peninsulares, edited by Esteban Krotz. Mérida: Programa de las Naciones Unidas para el Desarrollo, Universidad Autónoma de Yucatán.

Rossi, Luis Alejandro (2014). "Democracia Y Pluralismo En El Ocaso de La Soberanía." Araucaria 16 (31): 3-23. http://www.scopus.com/inward/record.url?eid=2-s2.084899925168\&partnerID=tZOtx3y1.

Sahuí Maldonado, Alejandro (2016). "La Igualdad En La Medida de La Calidad Democrática Una Revisión Crítica de La Propuesta de Leonardo Morlino." Revista Mexicana de Ciencias Políticas Y Sociales LXI (227): 273-94.

Serrano, Sandra (2017). "La Igualdad, La Universalidad Y La Constitución." In Cien Ensayos Para El Centenario. Constitución Política de Los Estados Unidos Mexicanos, Tomo 4: Estudios Políticos, edited by Pedro Salazar Ugarte, Francisco Alberto Ibarra Palafox, and Esquivel. Gerardo, 309-326. México: UNAM, Instituto de Investigaciones Jurídicas.

Tylor, Edward (1979). La Ciencia de La Cultura. Edited by J. S. Kahn. Barcelona: Anagrama.

Velasco, Juan Carlos (2009). "Democracia Y Deliberación Pública." Confluencia XXI. Revista de Pensamiento Político 6: 70-79. 